\title{
A Unified Approach to the Analysis of Multicarrier DS-CDMA over Nakagami- $m$ Fading Channels
}

\author{
Lie-Liang Yang and Lajos Hanzo
}

\author{
Dept. of ECS, University of Southampton, SO17 1BJ, UK. \\ Tel: +44-703-593 125, Fax: +44-703-594 508 \\ Email: llydecs.soton.ac.uk and lh@ecs.soton.ac.uk \\ http://www-mobile.ecs.soton.ac.uk
}

\begin{abstract}
A class of unified multicarrier DS-CDMA (MC DS-CDMA) schemes is defined and its performance is considered over multipath Nakagam $m$ fading channels. The spacing between two adjacent subcarriers of the unified MC DS-CDMA scheme is a variable, allowing us to gain insight into the effects of the spacing on the bit error rate (BER) performance of MC DS-CDMA systems. This unified MC DS-CDMA scheme includes the subclasses of multitone DSCDMA and orthogonal MC DS-CDMA as special cases. The optimum spacing of the MC DS-CDMA system required for achieving the minimum BER is investigated and the BER performance of the system having optimum spacing is evaluated. The resultant BER is compared with that of both multitone DS-CDMA and orthogonal MC DS-CDMA.
\end{abstract}

\section{INTRODUCTION}

Recently, a novel family of code-division multiple-access (CDMA) systems based on the combination of CDMA and orthogonal frequency division multiplexing (OFDM) schemes - which is referred to as multicarrier CDMA (MC-CDMA) - has drawn a lot of attention $[1,2,3,4,5]$. MC-CDMA is capable of supporting high data rate services over hostile radio channels. The modulated signal can be generated with the aid of the Fast Fourier Transform (FFT) at the cost of low receiver complexity.

In these MC-CDMA schemes, the transmitter of both multitone based direct-sequence CDMA [2] (multitone DS-CDMA) and that of orthogonal multicarrier DS-CDMA (MC DS-CDMA) [3] spreads the serial-to-parallel (S-P) converted data streams using a given spreading code in the time domain. In a multitone DS-CDMA system the subcarrier frequencies are chosen to be orthogonal harmonics of each other with minimum frequency separation among them before DS spreading [2], which is expressed as

$$
\int_{0}^{T_{s}} \cos \left(2 \pi f_{i} t+\phi_{i}\right) \cdot \cos \left(2 \pi f_{j} t+\phi_{j}\right) d t=0, \text { for } i \neq j,
$$

where $T_{s}$ represents the symbol duration of the multitone DS-CDMA signal, while $\left\{f_{i}\right\}$ and $\left\{\phi_{i}\right\}$ are the subcarrier frequencies and initial phases, respectively. Therefore, in multitone DS-CDMA systems the spacing $\Delta$ between two adjacent subcarrier frequencies is $\Delta=1 / T_{s}$. The subcarrier frequencies, hence, take the form of $f_{0}+i / T_{s}$ for

This work has been funded in the framework of the IST project IST-199912070 TRUST, which is partly funded by the European Union. The authors would like to acknowledge the contributions of their colleagues. $i=0,1, \ldots, U-1$, where $U$ is the number of subcarriers and $f_{0}$ is the fundamental frequency. By contrast, in an orthogonal MC DSCDMA system the subcarrier frequencies are chosen to satisfy the orthogonality condition with the minimum possible frequency separation after DS spreading [3], which can be expressed as

$$
\int_{0}^{T_{c}} \cos \left(2 \pi f_{i} t+\phi_{i}\right) \cdot \cos \left(2 \pi f_{j} t+\phi_{j}\right) d t=0, \text { for } i \neq j
$$

Eq.(2) implies that the spacing $\Delta$ between two adjacent subcarrier frequencies is $\Delta=1 / T_{c}$, where $T_{c}$ is the chip duration of the DS spreading codes and the subcarrier frequencies take the form of $f_{0}+$ $i / T_{c}$ for $i=0,1, \ldots, U-1$.

Let $N_{e}=T_{s} / T_{c}$ be the spreading gain of the DS-spread subcarrier signals. Furthermore, we assume that each subcarrier's modulated signal has the same 'null-to-null' bandwidth of $2 / T_{c}$. Then, it can be readily shown that the condition of (1) actually includes the orthogonality condition of both the multitone and that of the orthogonal MC DS-CDMA schemes. It can be also readily shown that the orthogonality condition of (1) is obeyed, whenever the spacing $\Delta$ takes the form of $\Delta=\lambda / T_{s}, \lambda=1,2, \ldots$, where $\lambda$ is referred to as the normalized spacing between two adjacent subcarriers. The MC DS-CDMA scheme belongs to the family of multitone DS-CDMA arrangements, if $\lambda=1$, while to the class of orthogonal MC DS-CDMA systems, if $\lambda=N_{e}$. Furthermore, there exists no overlap between the mainlobes of the modulated subcarrier signals after DS spreading, when $\lambda=2 N_{e}$, which is the bandwidth requirement of the MC DS-CDMA system proposed in [4].

Based on the above observations, both the multitone DS-CDMA system and the orthogonal MC DS-CDMA system can be viewed as a member of the class of unified multicarrier DS-CDMA systems having arbitrary subcarrier spacing of $\lambda \in\{1,2, \ldots$,$\} . Hence, the$ above unified MC DS-CDMA system model includes a number of specific MC DS-CDMA schemes. Furthermore, based on the analysis of this general model, the results generated can be extended to different MC DS-CDMA systems by simply varying a single parameter, namely $\lambda$. Finally, the subcarrier spacing $\lambda$ can be optimized according to specific design requirements tailored to the communication environments encountered, in order to achieve the optimum performance in terms of $\lambda$. Therefore, in this contribution the performance of the above unified MC DS-CDMA system is evaluated over the range of Nakagami- $m$ multipath fading channels [6]. 


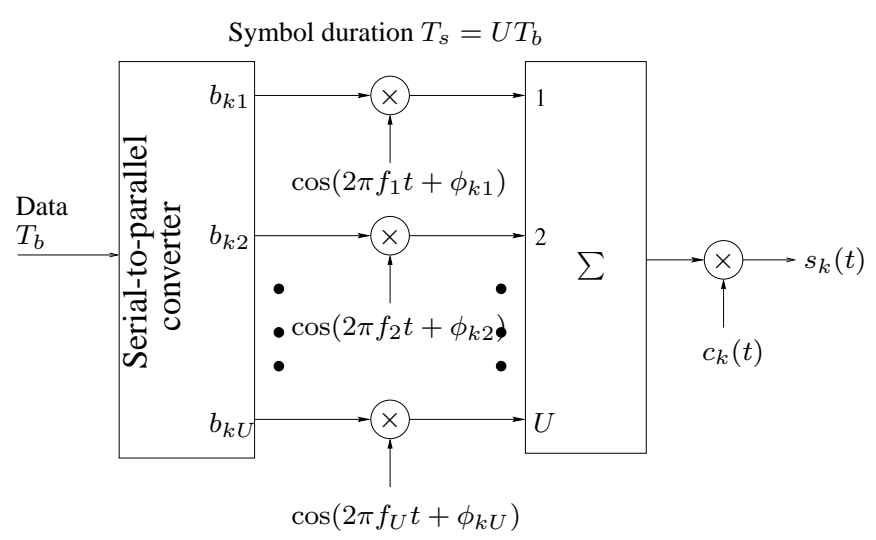

Figure 1: The $k$ th user's transmitter schematic for the unified MC DS-CDMA system.

\section{UNIFIED MULTICARRIER DS-CDMA SYSTEMS}

The transmitter schematic of the $k$ th user is shown in Fig. 1 for the unified MC DS-CDMA system. At the transmitter side, the binary data stream having a bit duration of $T_{b}$ is S-P converted to $U$ parallel sub-streams. The new bit duration of each sub-stream or the symbol duration is $T_{s}=U T_{b}$. After S-P conversion, the $u$ th sub-stream modulates a subcarrier frequency $f_{u}$ using Binary Phase Shift Keying (BPSK) for $u=1,2, \ldots, U$. As shown in Fig.1, after complexing the BPSK modulated sub-streams and spectral spreading, the transmitted signal of user $k$ can be expressed as

$$
s_{k}(t)=\sum_{u=1}^{U} \sqrt{2 P} b_{k u}(t) c_{k}(t) \cos \left(2 \pi f_{u} t+\phi_{k u}\right),
$$

where $P$ represents the transmitted power per subcarrier, while $\left\{b_{k u}(t)\right\}$, $c_{k}(t),\left\{f_{u}\right\}$ and $\left\{\phi_{k u}\right\}$ represent the data stream, the DS spreading waveform, the subcarrier frequency set and the phase angles introduced in the carrier modulation process. The data stream's waveform $b_{k u}(t)=\sum_{i=-\infty}^{\infty} b_{k u} P_{T_{s}}\left(t-i T_{s}\right)$ consists of a sequence of mutually independent rectangular pulses of duration $T_{s}$ and of amplitude +1 or -1 with equal probability. The spreading sequence $c_{k}(t)=\sum_{j=-\infty}^{\infty} c_{k j} P_{T_{c}}\left(t-j T_{c}\right)$ denotes the signature sequence waveform of the $k$ th user, where $c_{k j}$ assumes values of +1 or -1 with equal probability, while $P_{T_{c}}(t)$ is the rectangular chip waveform, which is defined over the interval $\left[0, T_{c}\right)$.

The spectrum of the unified MC DS-CDMA signal is shown in Fig.2, where $W_{s}=2 / T_{c 1}$ is the total bandwidth of the system, $T_{c 1}$ represents the chip-duration of a corresponding single-carrier DSCDMA signal, while $W_{d s}$ represents the 'null-to-null' bandwidth of the subcarrier signals. The spacing between two adjacent subcarrier frequencies is assumed to be $\Delta=\lambda / T_{s}$, where again, $\lambda$ indicates the normalized spacing. Finally, the subcarrier frequencies in Fig.2 are arranged according to $f_{u}=f_{0}+\frac{\lambda(1-U+u)}{2 T_{s}}, u=0,2,4, \ldots, 2(U-1)$.

Let $N_{e}=T_{s} / T_{c}$ be the spreading gain of the subcarrier signals in the unified MC DS-CDMA system and $N_{1}=T_{b} / T_{c 1}$ be the spreading gain of a corresponding single-carrier DS-CDMA system. Then, according to Fig.2, the system's total transmission bandwidth, the subcarrier spacing $\Delta$ and the DS spread bandwidth of the subcarrier signal obey the relationship of $W_{s}=(U-1) \Delta+W_{d s}$, or

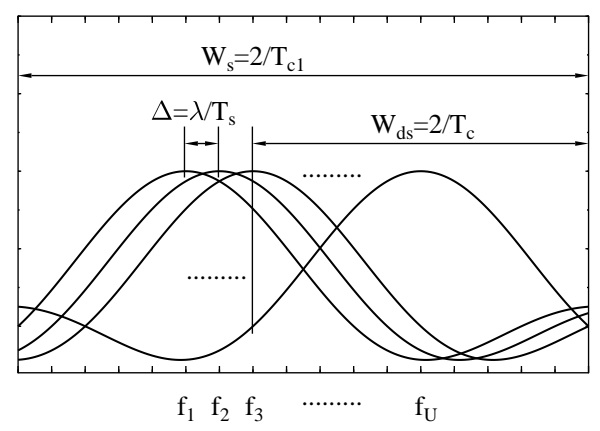

Figure 2: Stylized spectrum of the overlapping MC DS-CDMA signals.

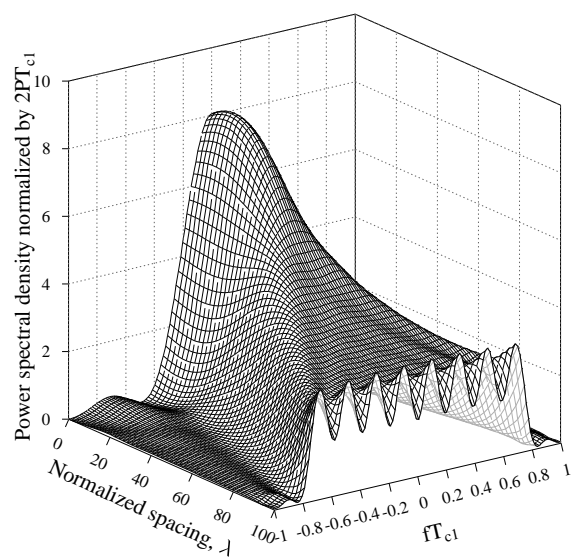

Figure 3: Baseband equivalent power spectral density of the transmitted signal seen in (3) in the unified MC DS-CDMA systems.

$$
\frac{2}{T_{c 1}}=(U-1) \frac{\lambda}{T_{s}}+\frac{2}{T_{c}} .
$$

Multiplying both sides of the above equation by the symbol duration, $T_{s}$, and taking into account that $T_{s}=N_{e} T_{c}$ as well as that $T_{s}=$ $U T_{b}=U N_{1} T_{c 1}$, the processing gain, $N_{e}$, of the subcarrier signals can be expressed as

$$
N_{e}=U N_{1}-\frac{(U-1) \lambda}{2},
$$

which implies that for a given total system bandwidth of $W_{s}=2 / T_{c 1}$ and for a given number of subcarriers, $U, N_{e}$ decreases, as $\lambda$ increases.

Fig. 3 shows the baseband equivalent power spectral density of the transmitted signal of (3) versus the normalized subcarrier spacing, $\lambda$, and the frequency-chip duration product, $f T_{c 1}$ for the parameters of $U=8, N_{1}=64$. The power spectral density surface shows that the transmitted signal's energy is concentrated at the center frequency, when the normalized spacing $\lambda$ is low, while spreads over the whole frequency band, when the normalized spacing is high. Furthermore, it can be seen that there exist normalized spacing values, for which the transmitted signal's energy is uniformly distributed over the available 
frequency band. Since the power spectral density of the unified MC DS-CDMA signals is a function of the spacing between the subcarriers, the power spectral density can be shaped online according to the system requirements, in order to maximize the spectrum efficiency in terms of the spacing $\lambda$ of the subcarriers.

We assume that the channel between the $k$ th transmitter and the corresponding receiver is a multipath Nakagami- $m$ fading channel [6] having $L_{p}$ number of resolvable paths. Let $T_{m}$ be the maximum delay-spread of the communication channel. Then, the number of resolvable paths, $L_{p}$, associated with the unified MC DS-CDMA signal is given by $L_{p}=\left\lfloor T_{m} / T_{c}\right\rfloor+1$ [4], where $\lfloor x\rfloor$ represents the largest integer not exceeding $x$. The number of resolvable paths, $L_{1}$, in the context of the corresponding single-carrier DS-CDMA signal is given by $L_{1}=\left\lfloor T_{m} / T_{c 1}\right\rfloor+1$. Multiplying both sides of (4) by $T_{m}$, we can see that $L_{p}$ and $L_{1}$ are related by

$$
L_{p} \approx\left\lfloor\frac{2 N_{e}\left(L_{1}-1\right)}{2 N_{e}+(U-1) \lambda}\right\rfloor+1 .
$$

Assuming $K$ asynchronous CDMA users in the system, where all of them use the same $U$ and $N_{e}$ values, the average power received from each user at the base station is also assumed to be the same, implying perfect power control. Consequently, the received signal can be expressed as

$$
\begin{array}{r}
r(t)=\sum_{k=1}^{K} \sum_{u=1}^{U} \sum_{l_{p}=0}^{L_{p}-1} \sqrt{2 P} \alpha_{u l_{p}}^{(k)} b_{k u}\left(t-\tau_{k l_{p}}\right) c_{k}\left(t-\tau_{k l_{p}}\right) \\
\cdot \cos \left(2 \pi f_{u} t+\varphi_{u l_{p}}^{(k)}\right)+n(t),
\end{array}
$$

where $\alpha_{u l_{p}}^{(k)}, \tau_{k l_{p}}$ and $\varphi_{u l_{p}}^{(k)}$ represent the attenuation factor, delay and phase-shift with respect to the $l_{p}$ th multipath component of the channel, respectively. We assume that both $\tau_{k l_{p}}$ and $\varphi_{u l_{p}}^{(k)}$ are uniformly distributed random variables over $\left[0, T_{s}\right)$ and $[0,2 \pi)$, respectively, while $\alpha_{u l_{p}}^{(k)}$ obeys the Nakagami-m distribution [6]. Furthermore, We assume a negative exponentially decaying multipath intensity profile (MIP) distribution given by $\Omega_{u l_{p}}^{(k)}=E\left[\left(\alpha_{u l_{p}}^{(k)}\right)^{2}\right]=$ $\Omega_{0} \exp \left(-\eta l_{p}\right), \quad \eta \geq 0$, where $\Omega_{0}$ is the mean signal strength corresponding to the first resolvable path and $\eta$ is the rate of average power decay. In (7) $n(t)$ represents the Additive White Gaussian Noise (AWGN) with zero mean and double-sided power spectral density of $N_{0} / 2$.

Let the first user be the user-of-interest and consider the correlatorbased RAKE receiver in conjunction with maximum ratio combining (MRC), as shown in Fig.4, where the superscript and subscript of the reference user's signal has been omitted for notational convenience. In Fig.4 $L, 1 \leq L \leq L_{p}$ represents the number of diversity branches used by the receiver. Assuming perfect estimation of each path's timedelay, attenuation and phase in the context of the reference signal, referring to Fig.4, after MRC the decision variable $Z_{v}$ of the 0th data bit corresponding to the $v$ th sub-stream of the reference user can be expressed as

$$
\begin{gathered}
Z_{v}=\sum_{l=0}^{L-1} Z_{v l}, v=1,2, \ldots, U, \\
Z_{v l}=\int_{\tau_{l}}^{T_{s}+\tau_{l}} r(t) \cdot \alpha_{v l} c\left(t-\tau_{l}\right) \cos \left(2 \pi f_{v} t+\varphi_{v l}\right) d t .
\end{gathered}
$$

Based on the decision variable $Z_{v}, v=1,2, \ldots, U$, the current data bit of the $v$ th sub-stream is decided to be 0 or 1 , depending on whether

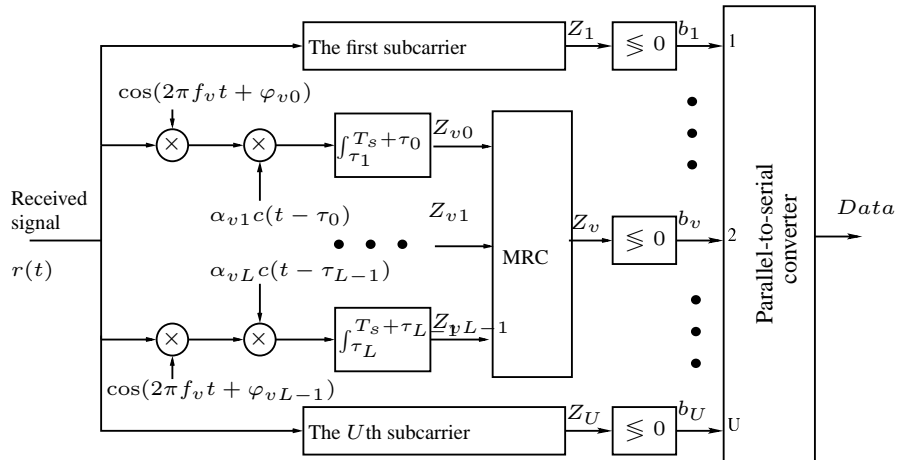

Figure 4: Receiver block diagram of the unified multicarrier DSCDMA system.

$Z_{v}$ is higher than zero. Finally, the $U$ number of parallel data substreams are parallel-to-serial converted, in order to output the serial data bits.

\section{BIT ERROR PROBABILITY}

In this section, the BER performance of the unified MC DS-CDMA system is analyzed with the aid of the Gaussian approximation for the decision variables of $Z_{v}, v=1,2, \ldots, U$. Based on the Gaussian approximation [2, 3], the BER using BPSK modulation conditioned on a set of fading attenuations $\left\{\alpha_{v l}, l=0,1, \ldots, L-1\right\}$ can be expressed as:

$$
P_{b}(\gamma)=Q\left(\sqrt{\frac{\left(E\left[Z_{v}\right]\right)^{2}}{\operatorname{Var}\left[Z_{v}\right]}}\right)=Q\left(\sqrt{2 \cdot \sum_{l=0}^{L-1} \gamma_{l}}\right)
$$

where

$$
\begin{aligned}
& \gamma_{l}= \gamma_{c} \cdot \frac{\alpha_{v l}^{2}}{\Omega_{0}} \\
& \gamma_{c}= {\left[\left(\frac{\Omega_{0} E_{b}}{N_{0}}\right)^{-1}+\frac{2\left(K L_{p}-1\right) q\left(L_{p}, \eta\right)}{L_{p}}\right.} \\
&\left.\cdot\left(\frac{1}{3 N_{e}}+(U-1) \bar{I}_{M}\right)\right]^{-1}
\end{aligned}
$$

and in (12) $q\left(L_{p}, \eta\right)=\left(1-e^{-\eta L_{p}}\right) /\left(1-e^{-\eta}\right)$, while $\bar{I}_{M}$ represents the average normalized variance of the multiuser interference inflicted by one path, which can be expressed as

$$
\begin{array}{r}
\bar{I}_{M}=\frac{1}{U(U-1)} \sum_{v=1}^{U} \sum_{\substack{u=1 \\
u \neq v}}^{U} \frac{N_{e}}{2 \pi^{2}(u-v)^{2} \lambda^{2}} \\
\cdot\left[1-\operatorname{sinc}\left(\frac{2 \pi(u-v) \lambda}{N_{e}}\right)\right] .
\end{array}
$$

Furthermore, in (10) the Gaussian $Q$-function has classically been defined as $Q(x)=\frac{1}{\sqrt{2 \pi}} \int_{x}^{\infty} \exp \left(-\frac{t^{2}}{2}\right) d t$. Alternatively, the Gaussian $Q$-function can be represented as [6]

$$
Q(x)=\frac{1}{\pi} \int_{0}^{\pi / 2} \exp \left(-\frac{x^{2}}{2 \sin ^{2} \theta}\right) d \theta, x \geq 0 .
$$


With the aid of (14) and using the PDFs of the instantaneous signal to noise (SNR), $\gamma_{l}$ for $l=0,1, \ldots, L-1$, the average BER can be written as

$$
P_{b}=\frac{1}{\pi} \int_{0}^{\pi / 2} \prod_{l=0}^{L-1} I_{l}\left(\bar{\gamma}_{l}, \theta\right) d \theta,
$$

where

$$
I_{l}\left(\bar{\gamma}_{l}, \theta\right)=\int_{0}^{\infty} \exp \left(-\frac{\gamma_{l}}{\sin ^{2} \theta}\right) p_{\gamma_{l}}\left(\gamma_{l}\right) d \gamma_{l} .
$$

Since $\gamma_{l}=\gamma_{c} \cdot \frac{\alpha_{m l}^{2}}{\Omega_{0}}$ and $\alpha_{m l}$ obeys the Nakagami- $m$ distribution [6], it can be shown that the PDF of $\gamma_{l}$ can be expressed as

$$
p_{\gamma_{l}}\left(\gamma_{l}\right)=\left(\frac{m}{\bar{\gamma}_{l}}\right)^{m} \frac{\gamma^{m-1}}{\Gamma(m)} \exp \left(-\frac{m \gamma_{l}}{\bar{\gamma}_{l}}\right), \gamma_{l} \geq 0,
$$

where $\bar{\gamma}_{l}=\gamma_{c} e^{-\eta l}$ for $l=0,1, \ldots, L-1$.

Upon substituting (17) into (16) it can be shown that

$$
I_{l}\left(\bar{\gamma}_{l}, \theta\right)=\left(\frac{m \sin ^{2} \theta}{\bar{\gamma}_{l}+m \sin ^{2} \theta}\right)^{m} .
$$

Finally, upon substituting (18) into (15), the average BER of the unified MC DS-CDMA system considered can be expressed as

$$
P_{b}=\frac{1}{\pi} \int_{0}^{\pi / 2} \prod_{l=0}^{L-1}\left(\frac{m \sin ^{2} \theta}{\bar{\gamma}_{l}+m \sin ^{2} \theta}\right)^{m} d \theta .
$$

In (19), since $\frac{m \sin ^{2} \theta}{\bar{\gamma}_{l}+m \sin ^{2} \theta}>1$, it can be readily shown that the average BER decreases exponentially, when increasing the value of $m$, i.e., when the Nakagami channel's fading is mitigated, while decreases approximately with the $l$ th power of $\frac{m \sin ^{2} \theta}{\bar{\gamma}_{l}+m \sin ^{2} \theta}$, if $\bar{\gamma}_{l}$ is sufficiently similar for different MIP index values of $l$.

\section{NUMERICAL RESULTS}

In this section we evaluate the performance of the unified multicarrier DS-CDMA scheme, investigate the effect of the normalized subcarrier spacing, $\lambda$, on the system's performance and compare the performance of MC DS-CDMA having the optimum spacing to that of both multitone DS-CDMA [2] and orthogonal multicarrier DS-CDMA [3]. Note that all the BER results in this section were derived from (19). For convenience, the parameters common in all figures are summarized as follows. The spreading gain and the number of resolvable paths of the corresponding single-carrier DS-CDMA system are $N_{1}=$ 128 and $L_{1}=32$, respectively. The MIP decay-factor is $\eta=0.2$. Throughout this section, the number of subcarriers used is $U=2,4,8$, and 32. The normalized subcarrier spacing of the multitone DSCDMA system is $\lambda=1$. According to (5) the normalized subcarrier spacing of the orthogonal MC DS-CDMA system using the above number of subcarriers becomes $\lambda=N_{e} \approx 170,204,227,240$ and 248 , respectively.

The influence of the normalized subcarrier spacing, $\lambda$, on the average BER of the unified MC DS-CDMA system is shown explicitly in Fig.5, where both the multipath Rayleigh fading channel associated with $m=1$ (Fig.5(a)) and the multipath Nakagami- $m$ fading channel of $m=20$ (Fig.5(b)) were considered. From the results of Fig.5(a) we infer that the BER of the multicarrier system using
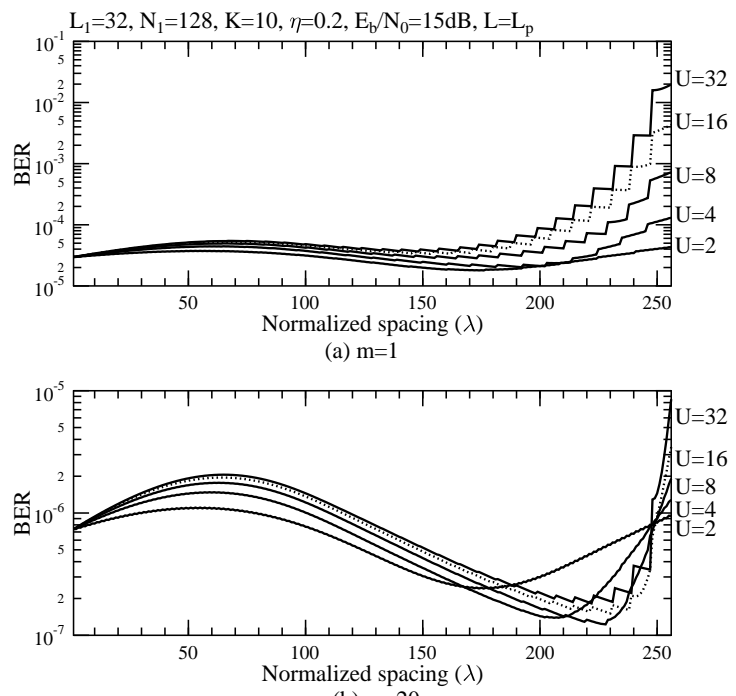

(b) $\mathrm{m}=20$

Figure 5: BER versus the normalized subcarrier spacing, $\lambda$ for the unified MC DS-CDMA system over the multipath Nakagami- $m$ fading channel.

a given number of subcarriers changes slowly, when $\lambda<200$, and there is no pronounced optimum spacing. In contrast to Fig.5(a), in Fig.5(b) the BER changes more explicitly, when changing the spacing $\lambda$. By carefully observing the curves in Fig.5(b), we find that the optimum spacing for a given value of $U$ was around $\lambda=N_{e}$, where the orthogonal MC DS-CDMA scheme achieves the best BER performance. In Fig.5(a) and Fig.5(b) we assumed that all the resolvable paths were combined in the receiver irrespective of the complexity imposed. Hence, the complexity of the RAKE receiver decreases, when increasing the spacing $\lambda$, since the number of resolvable paths, $L_{p}$, decreases for all values of $U$, when increasing the spacing, $\lambda$. Therefore, from this point of view, the multitone DS-CDMA scheme associated with $\lambda=1$ does not constitute a good design trade-off, when compared to the orthogonal MC DS-CDMA scheme having a spacing of $\lambda>>1$, since it results in a higher receiver complexity without any commensurate BER benefits. This is true even over the Rayleigh fading scenario of Fig.5(a), where the BER deficiency of the $\lambda=1$ scenario is less obvious.

In Fig. 6 the BER versus $E_{b} / N_{0}$ performance was evaluated in the context of the multitone, the orthogonal multicarrier and the 'spacing6 optimized' MC DS-CDMA schemes for $m=1,2$ and $\infty$. The system parameters employed were shown at the top of the figures. From these results we find that for $E_{b} / N_{0}>10.5 d B$, the multitone DSCDMA scheme outperforms the orthogonal MC DS-CDMA arrangement, but for any other cases, orthogonal MC DS-CDMA marginally outperforms multitone DS-CDMA. However, both the multitone and the orthogonal multicarrier DS-CDMA schemes are outperformed by the 'spacing-optimized' MC DS-CDMA scheme, when $m=1$ and 2. For the AWGN channel scheme of $m \rightarrow \infty$ the orthogonal and the 'spacing-optimized' MC DS-CDMA schemes achieve a similar BER performance across the entire SNR per bit range.

Finally, in Fig.7 we evaluated the BER versus the number of ac- 


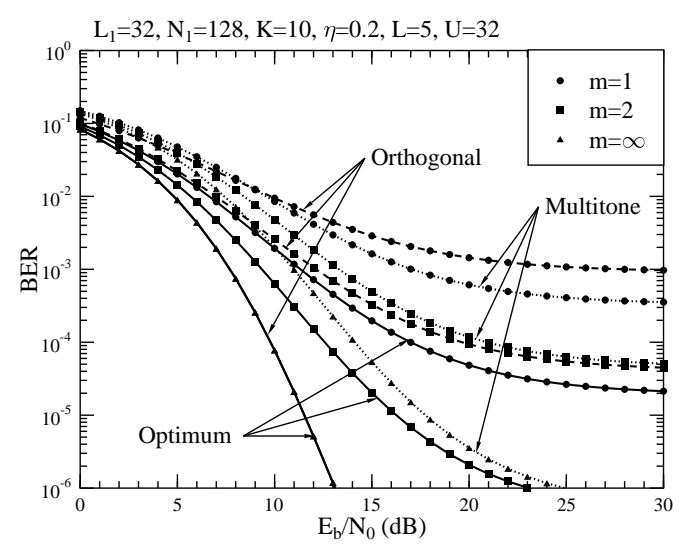

Figure 6: BER versus the SNR per bit, $E_{b} / N_{0}$, performance of the MC DS-CDMA system having the optimum normalized subcarrier spacing, for the multitone DS-CDMA and for the orthogonal MC DSCDMA systems over various multipath Nakagami- $m$ fading channels.

tive users, $K$, in the context of the multitone, as well as the orthogonal and 'spacing-optimized' MC DS-CDMA schemes in terms of $m=$ 1,2 and $\infty$. According to the results we observe that when $m=1,2$ and $K<20,6$, respectively, then multitone DS-CDMA outperforms orthogonal MC DS-CDMA. By contrast, for any other scenarios orthogonal MC DS-CDMA outperforms multitone DS-CDMA. However, both the multitone and the orthogonal MC DS-CDMA schemes are outperformed by the 'spacing-optimized' MC DS-CDMA scheme, when $m=1$ and 2. For the AWGN channel scenario of $m \rightarrow \infty$ the orthogonal multicarrier and the 'spacing-optimized' MC DS-CDMA schemes achieve a similar BER performance, regardless of the number of active users.

\section{REFERENCES}

[1] R. Prasad and S. Hara, "Overview of multicarrier CDMA," IEEE Communications Magazine, pp. 126-133, December 1997.

[2] L. Vandendorpe, "Multitone spread spectrum multiple access communications system in a multipath Rician fading channel," IEEE Transactions on Vehicular Technology, vol. 44, no. 2, pp. 327-337, 1995.

[3] E. A. Sourour and M. Nakagawa, "Performance of orthogonal multicarrier CDMA in a multipath fading channel," IEEE Transactions on Communications, vol. 44, pp. 356-367, March 1996.

[4] S. Kondo and L. B. Milstein, "Performance of multicarrier DS CDMA systems," IEEE Transactions on Communications, vol. 44, pp. 238-246, February 1996.

[5] L.-L. Yang and L. Hanzo, "Blind joint soft-detection assisted slow frequency-hopping multicarrier DS-CDMA," IEEE Transactions on Communications, vol. 48, pp. 1520 -1529, September 2000.

[6] M.-S. A. Marvin K. Simon, Digital Communication over Fading

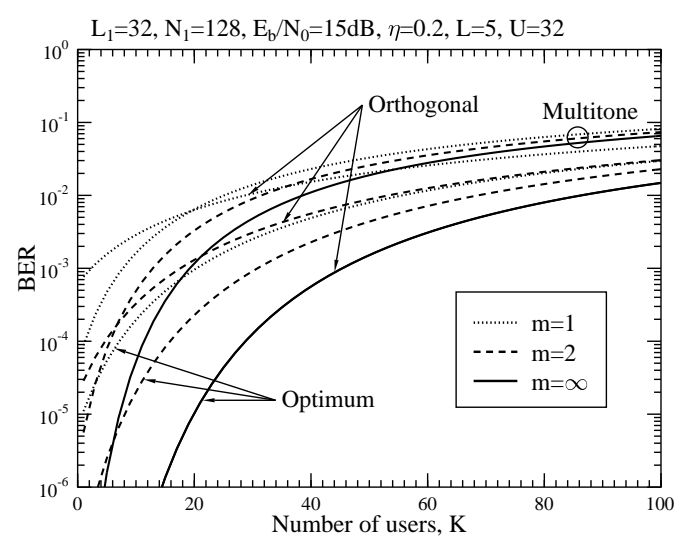

Figure 7: BER versus the number of users, $K$, for the MC DS-CDMA scheme having the optimum normalized subcarrier spacing, as well as for the multitone DS-CDMA and for the orthogonal MC DS-CDMA systems over various multipath Nakagami- $m$ fading channels.

Channels: A Unified Approach to Performance Analysis. New York: John Wiley \& Sons, 2000. 